during the study period. 708 (26.1\%) cases of diseases frequently associated with HIV were carried out. Suspected and treated pathologies were among other simple malaria in 262 cases (37\%), 177 cases of pulmonary diseases (25\%) including 03 cases of tuberculosis, 109 cases of gastroenteritis acute (15.4\%), 29 oral candidiasis and 29 sexual transmitted infections ( $4.1 \%$ each) and 4 cases of Herpes Zoster $(0.6 \%)$. For these diseases, the treatment success rate was $95.5 \%$. 16 hospitalizations have been made and 16 cases of consultations have been referred to physicians.

Conclusion The nurse can be a resource used to deal with the lack of physicians in management of PLHA especially if he received a specific training. This management training should be a key point in its basic studies curriculum in developing countries.

\section{P2.157 THE EXPERIENCE OF IMPLEMENTING POINT-OF-CARE HIV TESTING IN GP PRACTICES IN THE UNITED KINGDOM}

doi:10.1136/sextrans-2013-051184.0421

'S K Brooks, ${ }^{2}$ W Hachmoller. ${ }^{1}$ College of Agricultural Sciences and Education, Port Antonio, Portland, Jamaica; ${ }^{2}$ NHS North East London and the City, Newham Public Health Directorate, London, UK

Background NHS Newham implemented a General Practitioner (GP) rapid Point-of-care (PoCT) pilot for the testing of HIV in 2010. This was in response to recommendations made by the UK Chief Medical Officer and the British HIV Association for the expansion of routine HIV testing in all health care settings.

Methods Under the 12 month pilot, ten GP practises in Newham were recruited and trained to conduct rapid HIV testing, using the INSTI HIV-1/HIV-2 rapid testing kit. An HIV test was opportunistically offered to existing and new patients as routine. Accepting patients were tested following a pre-test discussion. Reactive antibody tests were referred to HIV clinical services.

Results During the pilot, a total of 698 tests were conducted in the participating GP practises. Of those tested, $58 \%$ were female and $37 \%$ were male. Eighteen percent (18\%) of clients tested were Black African. Greater up-take of HIV testing took place in the 25-34 year old age group. There were 11 reactive test results and 5 indeterminate results.

Conclusions Point-of-care (PoCT) testing of HIV is compatible in GP practises and acceptable to patients. Strategies are required for sustaining and expanding Point-of-care HIV testing in GP practises in London.

\section{P2.158 ONE-STOP SHOP SERVICE DELIVERY MODEL: INTEGRATING PREVENTION INTERVENTIONS WITH HIV CARE/ TREATMENT SERVICES IN A COMMUNITY-BASED MEDICAL HOME SETTING}

doi:10.1136/sextrans-2013-051184.0422

L Kudryashova Hernandez. Neighborhood Health Services Corporation, Plainfield, NJ, United States

Background Neighborhood Health Services Corporation (NHSC), an urban community-based not-for-profit ambulatory health centre in Plainfield, New Jersey, USA, provides services to over 350 persons living with HIV/AIDS. Presently $75 \%$ of NHSC HIV patients have history of substance abuse, $62 \%$ have mental health issues and/or depression and $30 \%$ are at risk for homelessness. For these patient populations long-term health and quality of life outcomes can not be achieved and sustained without aggressive intervention around substance use, mental health and other contributing factors.

Methods NHSC incorporates a coordinated, patient-centred approach to integrating multiple prevention services and interventions with HIV primary care and treatment in a welcoming, nonthreatening environment of a patient-centred medical home.
Substance abuse and mental health screenings are done by clinicians upon patient enrollment and every six months thereafter. Referrals for in-depth substance abuse and mental health assessments are generated as needed or at a minimum annually per clinical protocols. Patients in need of these services receive on-going counselling and appropriate interventions on-site. Referrals are also made to offsite facilities for crisis intervention and inpatient services. Psychosocial, financial and lifestyle assessments are conducted every six months to assess patients' risk for homelessness, substance use and unsafe lifestyle practises.

Results Resulting from an integrated, patient-centred approach to providing HIV services NHSC demonstrated the following: $95 \%$ of HIV patients received substance abuse and mental health screening; $100 \%$ received medical case management assessments. Furthermore, 57 patients receive on-going substance abuse counselling; 36 patients receive mental health counselling; 16 persons are in shelter/ transitional housing; 2 persons were hospitalised for suicide prevention.

Conclusions Integration of prevention interventions with HIV care/treatment under the umbrella of Early Intervention Services allowed to: achieve improved understanding of the reality of substance abuse/mental health; establish a seamless one-stop shop service delivery model and improve patients' access to community prevention/treatment resources.

\section{P2.159 KNOWLEDGE AND PRACTICE OF NURSES IN THE PREVENTION OF VERTICAL TRANSMISSION OF HIV IN SELECTED HOSPITAL OF EASTERN REGION OF NEPAL}

doi:10.1136/sextrans-2013-051184.0423

N Pokharel. B.P. Koirala institute of health sciences Dharan,Nepal, Dharan, Nepal

Vertical transmission remains the main mode of acquisition of HIV infection in children. A total of 700,000 children were newly infected in 2003, mainly through mother-to-child transmission of HIV. ${ }^{1}$ Mother to child transmission is also the largest source of HIV infection in children in Nepal. Nurses having the knowledge of HIV can bring positive changes in behaviour. Knowledge, training and experience in every aspect of one's profession are very important.

Objective The objective of this study is to assess knowledge and practise of Nurses in the Prevention of vertical transmission of HIV. Methods \& Materials:

Study Design A cross sectional study was done including 112 nurses drawn from the three selected hospital (BPKIHS Dharan, Koshi Zonal hospital Biratnagar and Mechi Zonal Hospital, Bhadrapur,) through population enumeration method.

Results The study show that half $(50.8 \%)$ of the respondents had adequate knowledge and near half (49.1\%) had inadequate level of knowledge, where total mean score \pm SD $28.3 \pm 5.5$. Only $16.1 \%$ of the respondents had good practise for prevention of vertical transmission of HIV. Qualification and place of working area are associated with knowledge of HIV/AIDS and PMTCT, where P value is $0.006,0.001$ respectively.

Conclusion In General, adequate knowledge was found half $(50.8 \%)$ of the respondents. Thus, more educational programmes should be focused on increasing their knowledge about vertical transmission, hoping to overcome the misconceptions that may be help to behaviour change toward safer practises.

\section{P2.160 UNDERAGE SEX - WHO CARES?}

doi:10.1136/sextrans-2013-051184.0424

A Delamere, A King, F Mulcahy. GUIDE Clinis, St James's Hospital, Dublin, Ireland

Background The Young Persons Clinic (YPC) was set up within the GUIDE Clinic as a dedicated clinic for young people aged 